\title{
AVALIAÇÃO DOS ERROS RADIOGRÁFICOS COMETIDOS POR ALUNOS DE GRADUAÇÃO DURANTE O TRATAMENTO ENDODÔNTICO
}

\author{
EVALUATION OF THE RADIOGRAPHIC ERRORS PERFORMED BY \\ UNDERGRADUATE STUDENTS DURING ENDODONTIC TREATMENT
}

\author{
Aletéia Massula de Melo Fernandes* \\ Alana Priscila Souza Aguiar* \\ Lorraine Pizzo da Cruz* \\ Marcos Uyeda Aivazoglou* \\ Ana Paula Martins Gomes** \\ Eduardo Galera da Silva** \\ Clóvis Pagani $\cdots$
}

\begin{abstract}
RESUMO
Introdução: O objetivo deste estudo é avaliar os erros radiográficos cometidos por alunos de graduação durante as diferentes fases do tratamento endodôntico. Métodos: Foram avaliadas 260 radiografias periapicais, divididas nos seguintes grupos: radiografia inicial, radiografia para odontometria, radiografia da prova do cone principal de guta-percha, radiografia da condensação lateral e radiografia final. As radiografias foram realizadas com o mesmo tipo de película (E-Speed - Kodak) e aparelho de Raios X (50KV, 7,5mA), tempo de exposição de 0,8 segundos e processadas pelo método visual. Com auxílio de lupa e negatoscópio, três examinadores avaliaram as radiografias e registraram erros relacionados à técnica ou processamento. Resultados: Os resultados mostraram que $57,70 \%$ das radiografias analisadas apresentaram algum tipo de erro (erro de técnica ou processamento). Não houve diferença estatística no número de radiografias com erros entre as diferentes fases do tratamento endodôntico. Conclusão: A maior frequência de erros ocorreu no quesito processamento radiográfico (manchamento, presença de riscos e densidade inadequada da imagem).
\end{abstract}

DESCRITORES: Radiografia dentária • Controle de qualidade • Diagnóstico.

\section{ABSTRACT}

Introduction: The aim of this study is to evaluate the radiographic errors performed by undergraduate students during the endodontic treatment. Methods: A total of 260 periapical radiographs were divided in these: groups initial radiography, length tooth, try-in of gutta-percha point, corroborative of the lateral condensation e final radiography. The radiographs were obtained employing the same kind of film (E-speed, Kodak) and of X-Ray units $(50 \mathrm{KV}, 7,5 \mathrm{~mA})$, with a 0,8 -second exposure time, and they were processed by visual method. With help of a magnifying glass and a viewbox, three examiners analyzed the radiographs and registered errors related to technique and processing. Results: The results showed that $57,70 \%$ of the radiographs analyzed exhibited some kind of errors (technique errors or processing). There were not significant differences in the number of the errors in the different phases of endodontic treatment. Conclusion: The highest frequency of errors was seen in radiograph processing (stains, presence of splines and inadequate radiographic density).

DESCRIPTORS: Radiography dental • Quality control • Diagnosis.

* Aluna(o) do Programa de Pós Graduação em Odontologia Restauradora, Faculdade de Odontologia de São José dos Campos - UNESP, SP, Brasil.

** Professora Adjunta da Disciplina de Endodontia, Departamento de Odontologia Restauradora, Faculdade de Odontologia de São José dos Campos - UNESP, SP, Brasil. e-mail: paula@fosjc.unesp.br

${ }^{* * *}$ Professor Assistente Doutor da Disciplina de Clínica Integrada, Departamento de Odontologia Social e Clínica Infantil, Faculdade de Odontologia de São José dos Campos - UNESP, SP, Brasil. e-mail: egaleras@terra. com.br

${ }^{* * * *}$ Professor Adjunto da Disciplina de Dentística, Departamento de Odontologia Restauradora, Faculdade de Odontologia de São José dos Campos - UNESP, SP, Brasil. e-mail: clovis@fosjc.unesp.br 


\section{N T RO DUÇÃ O}

A radiografia periapical é um exame complementar que fornece informações que não podem ser evidenciadas clinicamente, sendo utilizada pelos cirurgiõesdentistas durante o diagnóstico, planejamento, execução e proservação de um caso clínico no tratamento odontológico. No entanto, é necessário que as imagens radiográficas obtidas apresentem qualidade (Beldeman et al. ${ }^{1}, 1976$; Patel e Greer ${ }^{2}$, 1986; Helminen et al. $\left.{ }^{3}, 2000\right)$, que os pacientes sejam expostos a menor quantidade de radiação possível e que seja reduzido o número de radiografias insatisfatórias $\left(\right.$ Bean $^{4}, 1969$; Mourshed $^{5}$, 1971; Patel $^{6}$, 1979).

Falhas na execução das tomadas radiográficas, sejam elas de técnica ou de processamento, podem gerar interpretações errôneas, repetições, maior tempo de exposição dos pacientes à radiação, aumento do tempo clínico e dos custos do tratamento (Kreich et al. $\left.{ }^{7}, 2002\right)$. Além disso, a Organização Mundial de Saúde (OMS) mencionou que a baixa qualidade das imagens radiográficas, de maneira geral, pode reduzir a precisão do diagnóstico realizado a partir delas (Yacovenco $\left.{ }^{8}, 2001\right)$.

Certos erros cometidos durante a realização de uma radiografia são mais prováveis de ocorrer quando o operador não apresenta experiência suficiente para evitá-los ou quando ainda está em processo de aprendizagem. Assim, Patel e Greer ${ }^{2}$ (1986) concluíram, após a avaliação de 24.150 radiografias periapicais executadas por alunos, que são necessários de 20 a 25 exames radiográficos de boca completa para que o estudante possa adquirir uma proficiência técnica mínima. Porém, um grande esforço deve ser realizado durante o ensino das técnicas radiográficas pelos docentes para que o aluno atinja melhor desempenho em menor tempo.

Hoje, a Endodontia é uma das especialidades na clínica odontológica que mais utiliza radiografias periapicais, pois estas auxiliam o diagnóstico, são fundamentais durante a terapia endodôntica, principalmente nas fases de odontometria e obturação do canal radicular, e fornecem informações que ajudam na determinação do sucesso ou insucesso do caso após o término do tratamento. Porém, elementos como o isolamento absoluto e as limas endodônticas podem prejudicar o posicionamento e a manutenção do filme radiográfico, dificultando a técnica e favorecendo a obtenção de imagens de baixa qualidade (DeLyre e Johnson ${ }^{9}$, 1990).

Além disso, a grande limitação do exame radiográfico em fornecer uma imagem bidimensional de um objeto tridimensional, contribui como um fator de insucesso nos tratamentos endodônticos, uma vez que a identificação de canais mésio-vestibulares em molares pode ser prejudicada (Kulild e Peters ${ }^{10}$, 1990; Ramamurthy et al. ${ }^{11}$, 2006). Para solucionar esse problema, obedecendo ao princípio da paralaxe, pode-se realizar a variação do ângulo horizontal, permitindo a identificação de estruturas que estavam sobrepostas (Ramamurthy et al. ${ }^{11}, 2006$; Nance et al. ${ }^{12}$, 2000).

Assim, sabendo-se da importância de uma radiografia periapical de qualidade na Endodontia e entendendo que para corrigir ou diminuir os erros apresentados por alunos é necessário identificar as principais dificuldades encontradas por eles, o objetivo deste estudo é avaliar os erros radiográficos mais frequentemente cometidos por alunos de graduação durante a realização do tratamento endodôntico.

\section{MÉTODOS}

Esta pesquisa foi avaliada e aprovada pelo Comitê de Ética em Pesquisa sob número de protocolo 006/2009-PH/CEP, conforme Resolução 196/96 do Conselho Nacional de Saúde.

Para a realização da pesquisa, foram analisados 52 prontuários odontológicos, contendo as fichas clínicas e radiográficas dos pacientes atendidos na Disciplina de Endodontia de um Curso de Odontologia. Em cada prontuário analisado, encontravam-se cinco radiografias periapicais que foram divididas nos seguintes grupos: radiografia inicial, radiografia para odontometria, radiografia da prova do cone principal de guta-percha, radiografia da condensação lateral e radiografia final. Assim, 52 radiografias foram examinadas por grupo, fornecendo uma amostra total
FERNANDES AMM

AGUIAR APS

CRUZ LP

AIVAZOGLOU MU

GOMES APM

SILVA EG

PAGANI C.

AVALIAÇÃO

DOS ERROS

RADIOGRÁFICOS

COMETIDOS

POR ALUNOS

DE GRADUAÇÃO

DURANTE O

TRATAMENTO

ENDODONTICO
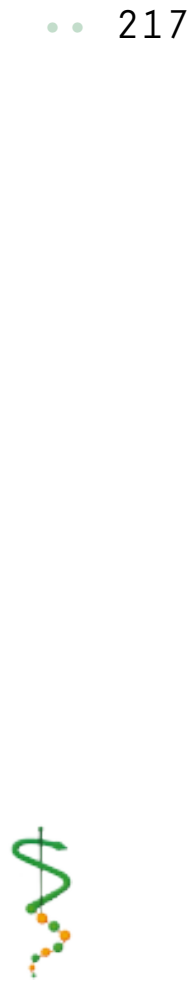

REVISTA DE ODONTOLOGIA DA UNIVERS I DADE CIDADE DE SÃO PAULO

$2010 ; 22(3): 216-$ 22, SET-DEZ 
FERNANDES AMM :

AGUIAR APS

$C R \cup Z L P$

AIVAZOGLOU MU

GOMES APM

SILVA EG

PAGANI C.

AVALIAÇÃO

DOS ERROS

RADIOGRÁFICOS

COMETIDOS

POR ALUNOS

DE GRADUAÇÃO

DURANTE O

TRATAMENTO

ENDODONTICO

\section{8}

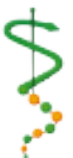

REVISTA DE

ODONTOLOGIA DA

UNI VERS I DADE

CIDADE DE SÃO

PAULO

$2010 ; 22(3): 216-$

22, SET-DEZ
ISSN 1983-5183

de 260 radiografias.

Todas as radiografias foram realizadas com o mesmo tipo de película radiográfica (E-Speed - Kodak) e aparelho de Raios X (Spectro 70X, Dabi Atlante), operando com 50KV, 7,5mA e tempo de exposição de 0,8 segundos. O processamento foi realizado em caixas acrílicas pelo método visual. Com auxílio de lupa (4X) e negatoscópio, três examinadores calibrados avaliaram as radiografias, levando em consideração os seguintes critérios:

PROCESSAMENTO:

- presença de manchas na radiografia (erro na lavagem final);

- presença de riscos na radiografia;

- densidade radiográfica: radiografias subprocessadas (claras) ou superprocessadas (escuras).

TÉCNICA:

- posicionamento incorreto do filme (corte do ápice ou região periapical);

- aparecimento da imagem do diafragma (meia lua ou halo);

- angulagem vertical (alongamento e encurtamento da imagem) e angulagem horizontal;

- filme invertido (radiografias realizadas com a película invertida em relação à sua face sensível).

Foram consideradas inadequadas todas as radiografias que apresentassem pelo menos um tipo de erro. Os dados obtidos foram submetidos aos testes de ANOVA e Tukey, dentro de um intervalo de confiança de 95\% (nível de significância para $\mathrm{p}<0,05)$. Para avaliar o nível de concordância entre os três examinadores, foi utilizado o índice de Kappa.

\section{RESULTADOS}

O índice de Kappa verificado entre os examinadores foi 0,88 , e indicou que houve concordância excelente entre os examinadores quanto aos resultados observados.

Das 260 radiografias periapicais analisadas, $110 \quad(42,30 \%)$ mostraram-se adequadas, ou seja, não apresentaram nenhum tipo de erro de técnica ou processamento. Foram verificados 222 erros distribuídos nas 150 radiografias restantes $(57,70 \%)$, com média de 1,48 erros por radiografia inadequada. A Tabela 1 mostra o número de erros radiográficos encontrados nas 150 radiografias inadequadas, de acordo com o tipo de erro e indicação da radiografia.

O teste de ANOVA a dois fatores mostrou diferenças significantes apenas para o fator tipo de erro radiográfico, como pode ser verificado na Tabela 2 . O teste de Tukey para o fator tipo de erro radiográfico apontou o grupo "manchamento" como diferente estatisticamente de todos os demais, não havendo diferenças entre os grupos "posicionamento incorreto do filme", "filme invertido", "imagem do diafragma" e "ângulo vertical ou horizontal". Também não houve diferença entre os grupos "densidade radiográfica" e "presença de riscos", como mostra a Tabela 3.

O tipo de erro mais encontrado foi o manchamento, seguido pela presença de riscos e erro de densidade radiográfica.

\section{I SCUSSÃO}

É importante ressaltar a importância de estudos como este, que investigam erros cometidos por alunos durante o curso de graduação, pois auxiliam a reconhecer as maiores dificuldades dos estudantes no decorrer do processo de aprendizagem e estimulam os docentes a buscar melhores recursos ou métodos para solucionar as deficiências apresentadas.

Das 260 radiografias examinadas, observou-se que 150 apresentaram erros radiográficos, correspondendo a 57,70\% do total. Nesse contexto, estão englobados os erros cometidos durante a técnica radiográfica e o processamento da imagem, sendo que o processamento apresentou as maiores médias de erros. Esses resultados mostraram que a obtenção de uma radiografia periapical satisfatória é um procedimento de difícil execução para um aluno de graduação no início da clínica odontológica, já que aproximadamente metade dos exames realizados foi considerada inadequada e teriam que ser repetidos, aumentando os custos do tratamento odontológico e o tempo clínico.

Ao comparar-se as médias obtidas com as observadas em outros estudos, pôde-se verificar que os resultados desta pesquisa foram melhores que os obtidos por Ma- 
theus et al. ${ }^{13}$ (2000) e por Gasparini et al. ${ }^{14}$ (1992) que apresentaram, respectivamente $90,1 \%$ e $91,5 \%$ de radiografias consideradas insatisfatórias devido à presença de erros de técnica e de processamento. Estudos mais antigos não consideraram erros de processamento e por isso mostraram porcentagens maiores de radiografias adequadas, como verificado nos trabalhos de Crandell ${ }^{15}$ (1958) e Bean ${ }^{4}$ (1969). Porém, é conveniente esclarecer que corrigir os erros cometidos durante o processamento é tão importante quanto a correção dos erros de técnica, já que uma radiografia mal processada pode inviabilizar a sua interpretação. Mesmo que se consiga visualizar a imagem radiográfica após o processamento incorreto, esta provavelmente não se manterá visível a longo prazo, devido principalmente ao aparecimento de manchas, impossibilitando o acompanhamento radiográfico de um caso clínico ao longo dos anos.

Não houve diferença estatisticamente significante no número de erros radiográficos encontrados nas diferentes fases do tratamento endodôntico (tipo de radiografia analisado). Porém, verifica-se que as radiografias iniciais e finais apresentaram menor quantidade de erros, o que pode ser justificado pela maior facilidade de execução da técnica radiográfica sem o aparato endodôntico (limas e isolamento absoluto).

Em relação aos tipos de erros radiográficos estudados, observou-se que os mais frequentes foram o manchamento, a presença de riscos e a densidade radiográfica, apresentando diferenças estatisticamente significantes em relação aos outros erros analisados. Assim, pôde-se verificar que as maiores médias de erros ocorreram durante o processamento da radiografia. Esses resultados foram semelhantes aos verificados por Silva et al. ${ }^{16}$ (2006) e Felippe et al. ${ }^{17}$ (2009). Silva et al. ${ }^{16}$ (2006) observaram que os erros de processamento foram responsáveis por $16 \%$ das repetições radiográficas, enquanto $84 \%$ ocorreram em outras etapas do processo radiográfico. Dentre os erros de processamento, $46 \%$ das radiografias foram repetidas devido à presença de riscos, $6 \%$ por falha na lavagem final, $35 \%$ por estarem claras ou escuras, $10 \%$ por velamento e $3 \%$ por não reposição de químicos. Felippe et al. ${ }^{17}$ (2009) observaram que a maior frequência de erros ocorreu no quesito processamento, seguido pelo posicionamento e contraste da imagem (imagens claras foram mais frequentes que imagens escuras).

Com os avanços tecnológicos e o aprimoramento da radiografia digital, os erros de processamento radiográfico podem ser minimizados, uma vez que essa técnica permite correções e melhoria da imagem, além do armazenamento direto no computador (Tsesis et al. ${ }^{18}$, 2008). Porém, devido ao alto custo, esse recurso não se encontra disponível em todas as Universidades para utilização por alunos de graduação. Além disso, alguns estudos relatam não haver diferença estatisticamente significante na utilização de imagens digitalizadas quando comparadas com as convencionais na prática clínica (Tsesis et al. ${ }^{18}$, 2008; Kositbowornchai et al. ${ }^{19}, 2001$ ).

Assim, os resultados deste estudo mostram que é preciso conscientizar os alunos sobre a importância do processamento radiográfico, esclarecendo de forma mais enfática todos os passos necessários para que isto ocorra, e buscando resultados práticos ao término do ano letivo. Dessa forma, todas as radiografias deveriam ser adequadamente obtidas e processadas, minimizando-se os custos do tratamento e possibilitando a manutenção adequada dos prontuários odontológicos.

\section{CONCLUSÃO}

De acordo com os resultados do presente trabalho, concluiu-se que.

Entre as radiografias analisadas, $57,70 \%$ apresentaram algum tipo de erro radiográfico, sendo necessário reforço dos conceitos de técnica e processamento durante os procedimentos clínicos.

Não houve diferença estatística no número de radiografias com erros nas diferentes fases do tratamento endodôntico;

Os erros mais frequentes foram o manchamento, a presença de riscos e a densidade radiográfica, tendo estas falhas ocorridas durante o processamento radiográfico.
FERNANDES AMM

AGUIAR APS

CRUZ LP

AIVAZOGLOU MU

GOMES APM

SILVA EG

PAGANI C.

AVALIAÇÃO

DOS ERROS

RADIOGRÁFICOS

COMETIDOS

POR ALUNOS

DE GRADUAÇÃO

DURANTE O

TRATAMENTO

ENDODONTICO

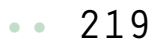

$\therefore 219$

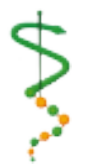

REVISTA DE ODONTOLOGIA DA UN I VERS I DADE Cidade de São PAULO

$2010 ; 22(3): 216-$ 22, SET-DEZ 
I S SN $1983-5183$

FERNANDES AMM AGUIAR APS

$C R \cup Z L P$

AIVAZOGLOU MU

GOMES APM

SILVA EG

PAGANI C.

AVALIAÇÃO

DOS ERROS

RADIOGRÁFICOS

COMETIDOS

POR ALUNOS

DE GRADUAÇÃO

DURANTE O

TRATAMENTO

ENDODONNTICO

Tabela 1 - Número de erros radiográficos encontrados nas 150 radiografias inadequadas, de acordo com o tipo de erro e indicação da radiografia

\begin{tabular}{|c|c|c|c|c|c|c|c|}
\hline Tipo de erro & $\begin{array}{l}\text { Rad. } \\
\text { Inicial }\end{array}$ & $\begin{array}{l}\text { Rad. } \\
\text { Odontometria }\end{array}$ & $\begin{array}{l}\text { Rad. } \\
\text { Cone } \\
\text { principal }\end{array}$ & $\begin{array}{l}\text { Rad. } \\
\text { Condensação } \\
\text { lateral }\end{array}$ & Rad. Final & $\begin{array}{l}\text { Total de } \\
\text { erros }\end{array}$ & Média \\
\hline $\begin{array}{l}\text { Posic. incorreto } \\
\text { do filme }\end{array}$ & 0 & 2 & 1 & 1 & 1 & 5 & 0,03 \\
\hline Densidade & 9 & 17 & 7 & 9 & 5 & 47 & 0,31 \\
\hline Filme invertido & 0 & 0 & 1 & 0 & 0 & 1 & 0,006 \\
\hline Manchamento & 7 & 20 & 22 & 24 & 13 & 86 & 0,57 \\
\hline $\begin{array}{l}\text { Imagem do } \\
\text { diafragma }\end{array}$ & 0 & 2 & 0 & 1 & 1 & 4 & 0,02 \\
\hline Riscos & 5 & 12 & 15 & 10 & 6 & 48 & 0,32 \\
\hline $\begin{array}{c}\text { Ang. Vertical ou } \\
\text { Horizontal }\end{array}$ & 7 & 8 & 6 & 5 & 5 & 31 & 0,20 \\
\hline Total de erros & 28 & 61 & 52 & 50 & 31 & 222 & 1,48 \\
\hline Média & 0,18 & 0,40 & 0,34 & 0,33 & 0,20 & 1,48 & \\
\hline
\end{tabular}

Tabela 2 - ANOVA a 2 fatores para o tipo de erro radiográfico e indicação da radiografia

\begin{tabular}{cccc}
\hline \hline Fatores & Grau de liberdade & $\mathrm{F}$ & $\mathrm{p}$ \\
\hline Tipo de erro & 7 & 20,71 & $0,000^{*}$ \\
Indicação da radiografia & 4 & 2,66 & 0,054 \\
\hline \hline
\end{tabular}

* $p<0,05$ - diferença estatisticamente significante

Tabela 3 - Resultados do Teste de Tukey (5\%) para o fator tipo de erro radiográfico

\begin{tabular}{ccc}
\hline \hline Tipo de erro & Média & Conjuntos $^{\text {Homogêneos }}{ }^{*}$ \\
\hline Posicionamento incorreto do filme & 0,03 & $\mathrm{~A}$ \\
Filme invertido & 0,006 & $\mathrm{~A}$ \\
Imagem do diafragma & 0,02 & $\mathrm{~A}$ \\
Ang. Vertical ou horizontal & 0,20 & $\mathrm{~A}$ \\
Densidade & 0,31 & $\mathrm{~B}$ \\
Riscos & 0,32 & $\mathrm{~B}$ \\
Manchamento & 0,57 & $\mathrm{C}$ \\
\hline \hline
\end{tabular}

* Letras iguais na mesma coluna não apresentam diferenças estatisticamente significantes 
FERNANDES AMM

Aguiar aPS

CRUZ LP

AIVAZOGLOU MU

GOMES APM

SILVA EG

PAGANI C.

AVALIAÇÃO

DOS ERROS

RADIOGRÁFICOS

cOMETIDOS

POR ALUNOS

de gRADUAÇão

DURANTE O

TRATAMENTO

ENDODONTICO

5. Mourshed F. A study of intraoral radiographic errors made by dental students. Oral Surg Oral Med Oral Pathol 1971 Nov; 32(5): 824-8.

6. Patel JR. Intraoral radiographic errors. Oral Surg Oral Med Oral Pathol 1979 Nov; 48(5): 479-83.

7. Kreich EM, Queiroz MGS, Sloniak MC. Controle de qualidade em radiografias periapicais obtidas no curso de Odontologia da UEPG. Publicatio UEPV: Biological and Health Sciences 2002; 8(1):33-45.

8. Yacovenco A. Análise dos problemas da radiografia na prática odontológica. Revista ABRO 2001 Jan-Jun; 2(1): 29-39.

9. DeLyre WR, Johnson ON Thomson EM. The periapical examination. In: Essentials of dental radiography for dental assistants and higienists. Conecticut: Appleton \& Lange. 1990.p.683-4.

10. Kulild JC, Peters DD. Incidence and configuration of canal systems in the mesiobuccal root of maxillary first and second molars. J Endod 1990 Jul; 16(7): 311-7.

11. Ramamurthy R, Scheetz JP, Clark SJ, Farman AG. Effects of imaging system and exposure on accurate detection of the second mesio-buccal canal in maxillary molar teeth. Oral Surg Oral Med Oral Pathol Oral Radiol Endod 2006 Dec; 102(6): 796802.

12. Nance R, Tyndall D, Levin LG, Trope M. Identification of root canals in molars by tuned-aperture computed tomography. Int Endod J 2000 Jul; 33(4): 392-6.

13. Matheus RA, Montebelo Filho A, Tanaka EE, Barros RM. Avaliação no desempenho de acadêmicos durante tomadas radiográficas periapicais, pela técnica da bissetriz, realizada na Faculdade de Odontologia da Universidade Norte do Paraná (Unopar). RFO UFP 2000 jul - dez; 5(2): 25-9.

14. Gasparini D, Vaz EMS, Haiter Neto F, Boscolo FN. Análise de erros radiográficos cometidos por alunos da Faculdade de Odontologia de Piracicaba no período de 1975 a 1988. Rev Odont USP 1992 jul-dez; 6(3/4): 107-14.

15. Crandell DE. Cause and frequency of intra-oral X-ray errors by dental and hygiene students. J Dent Educ 1958;22:189-96.

16. Silva PRD, Gambier VCR, Dias LPT, Lascala CA. Estudo sobre os erros mais comuns no processamento radiográfico. Rev Assoc Paul Cir Dent 2006 mar-abr; 60(2): 1125 .

17. Felippe MCS, Nassri MRG, Burgos PG, Freitas SFT, Lage-Marques JL. Quality of periapical radiographs taken by undergraduate students during endodontic treatment. RSBO 2009; 6(1): 63-9. 
FERNANDES AMM AGUIAR APS $C R \cup Z L P$ AIVAZOGLOU MU GOMES APM SILVA EG PAGANI C.

AVALIAÇ̃̃O DOS ERROS RADIOGRÁFICOS COMETIDOS POR ALUNOS DE GRADUAÇÃO DURANTE O TRATAMENTO ENDODONNTICO
18. Tsesis I, Kamburoglu K, Katz A, Tamse A, Kaffe I, Kfir A. Comparison of digital with conventional radiography in detection of vertical root fractures in endodontically treated maxillary premolars: an ex vivo study. Oral Surg Oral Med Oral Pathol Oral Radiol Endod 2008 Jul;106(1): 124-8.

19. Kositbowornchai S, Nuansakul R, Sikram S, Sinahawattana S, Saengmontri S. Root fracture detection: a comparison of direct digital radiography with conventional radiography. Dentomaxillofac Radiol 2001 Mar; 30(2): 106-9.

Recebido em: 11/09/2009

Aceito em: 9/08/2010. 